\title{
Optimization of resource demand for passenger services at airports during system failures such as blackouts
}

\author{
Lisa-Marie Brause $^{1^{*}}$ (D), Andrei Popa ${ }^{1}$, Tobias Koch², Andreas Deutschmann ${ }^{1}$ and Martin Hellmann ${ }^{3}$
}

\begin{abstract}
Background: In recent years, power outages at airports caused total collapses of terminal operations at the affected airports. These incidents have shown that existing emergency power systems were not sufficient to maintain airport operation. To mitigate the consequences of power outages, it is necessary to improve the management of available resources.

Methodology: Therefore, we investigate the possibility to save resources associated to passenger handling processes before departure. For this purpose, we create a simulation model of a generic medium-sized commercial airport. Several simulations with a varying number of service stations at check-in, security and passport control are carried out. Subsequently, we analyze the number of passengers who do not reach their flight due to increased waiting times at the passenger handling services.
\end{abstract}

Results: The simulations show that a reduction in resources at check-in counters and passport control initially has no effect on the number of passengers who do not reach their flight. The security control represents a bottleneck in passenger handling. In view of a break-even seat load factor the simulation results show, that a reduction of resources at the check-in below 16 counters, at the security check below 21 and at the passport control below 3 service stations should be avoided.

Conclusion: Therefore, the developed methodology is able to provide airport operators with decision support during system failures, as to which and how many service stations can be dispensed within the sub-processes of passenger handling without interrupting flight operations.

Keywords: Airport, Passenger simulation, Passenger handling, Power outage, Risk management, Resource optimization

\section{Introduction and state-of-the-art}

Within the last years air traffic gained more and more importance due to its advantages such as safety, punctuality, speed and reliability. Therefore, people, but also economy and politics, became dependent on its undisruptive functionality $[1,2]$. Nevertheless, past incidents have shown that system outages at airports caused total collapses of the operation at the concerning airports. In fact, safety measures like emergency power systems exist, which shall mitigate consequences. However, these were

\footnotetext{
* Correspondence: Lisa-Marie.Brause@dlr.de

${ }^{1}$ German Aerospace Center (DLR), Institute of Air Transport and Airport

Research Lilienthalplatz 7, 38108 Braunschweig, Germany

Full list of author information is available at the end of the article
}

insufficient to maintain a safe and secure air transport [3]. As an example, in December 2017, the passenger handling of the Hartsfield-Jackson Airport in Atlanta was interrupted due to a fire in the electric installation of the airport. Therefore, more than 1000 flights had been cancelled. Following this incident, a press officer of the Arbeitsgemeinschaft Deutscher Verkehrsflughäfen (ADV) declared that German airports are well prepared against power outages. German airports are provided by a redundant power system support, which enables continuous operation for several hours [4]. The power outage at the German airport in Hamburg outlines that this is questionable. On 3rd June 2018 a short circuit occurred in the electric network of the airport and caused

\section{Springer Open}

(0) The Author(s). 2020 Open Access This article is licensed under a Creative Commons Attribution 4.0 International License, which permits use, sharing, adaptation, distribution and reproduction in any medium or format, as long as you give appropriate credit to the original author(s) and the source, provide a link to the Creative Commons licence, and indicate if changes were made. The images or other third party material in this article are included in the article's Creative Commons licence, unless indicated otherwise in a credit line to the material. If material is not included in the article's Creative Commons licence and your intended use is not permitted by statutory regulation or exceeds the permitted use, you will need to obtain permission directly from the copyright holder. To view a copy of this licence, visit http://creativecommons.org/licenses/by/4.0/. 
an organizational shutdown during the whole day. Even though there must have been power supply due to the emergency power generator, airport operation could not be maintained. About 200 flights had been cancelled and thousands of passengers were hindered from continuing their journey [5].

These occurrences emphasize, that it is essential to improve the reaction to system failures. Though, it is not always possible to prevent system failures, we may mitigate their consequences. An important component is the allocation of resources for passenger handling. Even during system outages security and safety regulations at airports e.g. for security or passport control must be complied continuously. Moreover, passenger handling processes such as check-in or baggage handling also have to be maintained in order to enable an orderly flow of airport operation. Therefore, it is essential to improve the management of resources and to distribute the available power to all essential processes in an optimal manner. To investigate possible solutions without influencing daily operations of the airport, mathematical models or simulations may be used.

Mathematical models formally represent a decision or planning problem to determine solution proposals with the help of suitable procedures. As an example, Koch et al. used linear algebra to relate the impact of system state changes at airports, e.g. due to power shortages, to the process of passenger handling in the airport terminal [6]. In addition, Bruno et al. and Lalita et al. used mathematical models to improve the management of the check-In process at the airport terminal [7, 8].

Computer based simulations are a common tool to investigate processes in complex systems. Simulations of passenger flow at airports already have been performed successfully. In 2008, Fraport AG simulated the passenger flow at Frankfurt airport in order to improve current passenger flow and waiting times. As a result, an increased number of passengers could be handled without upcoming problems or constructional measures [9]. Furthermore, simulation models of airports can be used to analyze system performances under different conditions. In 2007, the Airport Lamezia Terme in Calabria (Italy) was simulated in order to analyze the average waiting times of passengers before reaching the gate under different conditions i.e. different availability of resources or passenger behavior. The results of the simulation study were compared to real data. Curcio et al. were able to show that the simulation data correspond to the real data. Therefore, simulation models are considered suitable to simulate airport terminal processes [10]. In addition, simulations are a common method in other parts of transport research. As an example, Asmer et al. investigated the possibility to install security checks at train stations by means of simulations [11]. In all studies AnyLogic was used as the simulation software.
As the airport terminal is a complex system involving multiple stakeholders and interactions, there are lots of research projects that developed models to investigate different aspects of an airport terminal [12]. However, recent work can be divided into four main topics: capacity planning $[13,14]$, operational planning and design $[15,16]$, security policy and planning [17] and airport performance review [18] [19]. Though, this work mainly treats the optimization of single process parameters such as waiting times at check-in [14] or security control [20]. However, since the processes influence each other, a holistic approach is required.

In this study, we use AnyLogic to model a generic airport and optimize its performance under exceptional circumstances such as power shortages. Thus, we simulate the effect of lower resource availability for the passenger handling services in the terminal before departure, consisting of check-in, baggage control, security checks and passport control. Our results provide information on the amount of passenger, who do not reach their flight in time due to increased waiting times at the passenger handling services. Using a break-even seat load factor we are able to evaluate the allocation of the resources as sufficient, critical and insufficient to maintain airport operation.

\section{Concept and simulation}

Our aim is to improve resource management at the airport terminal during system failures, so that flight operation can be maintained even during power shortages.

Figure 1 shows the connection of the system operating condition to the appropriate type of control regime i.e. the required reactive measure by the operator. While under normal system conditions (reaching from green to yellow in Fig. 1) measures are only required to optimize system state, in stability or viability state reactive measures are essential to keep operation viable. Within this study, we aim to determine the measures most applicable to keep system operation going.

This planning problem can be described by eq. 1 . Resources at the passenger handling services are minimized while the resulting seat load factor (SLF) must not fall under a given break-even SLF.

$$
\min (\text { resources } \mid \text { slf }>\text { break even slf })
$$

To solve this optimization problem, we develop a simulation model and conduct a parameter study. Gradually we minimize the resources at check-in, security check and passport control to determine an approximate solution. Evaluating the output data of all simulations we are able to find a minimum configuration of the process stations depending on the power supply of the airport. This provides airport operators with decision 


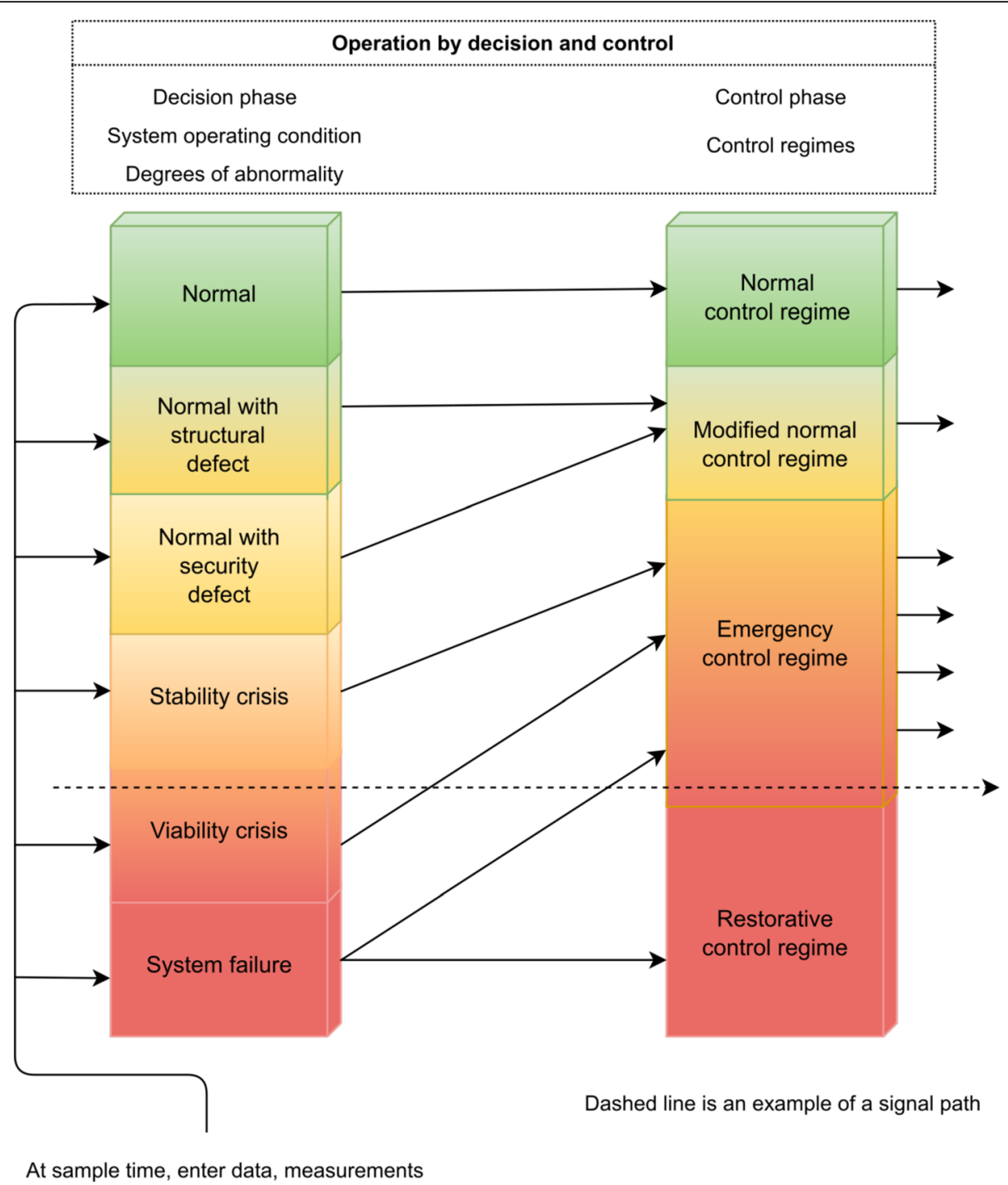

Fig. 1 Dependence of the appropriate decision and control regime on the system operating condition after llić and Zaborski [21] as depicted by Koch et al. [22]

support during system failures, as to which and how many service stations can be dispensed within the subprocesses of passenger handling without interrupting flight operations. In this paper, we only consider the passenger handling process for departures. The process for arriving passengers and the baggage handling processes are neglected due to a lack of information that is open to the public.

\subsection{Simulation model}

For the investigation, a model of a generic medium-sized commercial airport was developed, which is based on components of real airports. The advantage of a generic model is the flexibility i.e. the results can be transferred to all medium sized airports. Because of the amount of data open to the public, these components were taken from Cologne-Bonn Airport and are based on public information. Due to the international standards for airport operations, e.g. those set by the ICAO Annex 9 [23] or the European Commission [24], we can assume the data is transferable to other medium sized airports.

The passengers are modelled individually, but their behavior is subject to statistical distribution functions regarding baggage claim or check-in at the airport terminal. Interactions between passengers are only partially taken into account i.e. at the process stations only one passenger can be handled at a time and the passengers have to wait in queues for a free service station. However, passenger interactions between the process stations are neglected due to the assumption, that the dimension of the terminal is sufficient to avoid passenger congestion. 
Figure 2 shows the simulation logic of the model. The passengers are generated at the terminal entrance, according to their arrival time at the airport. Passengers, who did not check-in online in advance or have to check-in their baggage, proceed to the check-in counters. The number of the counter depends on the airline company the passenger is flying with. If the passenger travels with hand luggage only, he can proceed straight to the security control. Within the model, we only distinguish between these two types of check-in. Automatic check-in is not offered at all airports or by all airlines and is therefore neglected. Passengers who already checked-in the evening before or at stations outside the terminal do not differ from passengers, who checked-in online. Therefore, they are not considered separately. Moreover, the model simplifies the assumption that the baggage can only be checked-in at the check-in counters. Thus, separate check-in counters for baggage or vending machines, as well as counters for special or bulky luggage are neglected. The check-in counters open $2 \mathrm{~h}$ and close $30 \mathrm{~min}$ before departure [25]. Passengers arriving later at the check-in counter miss their flight. These passengers do not pass through processes in the airport terminal and directly leave the airport i.e. they are removed from the simulation.

The model includes a central security checkpoint with several security lanes, which all passengers have to pass, independently of their flight. At each control lane, four passengers can deposit and pick up their personal belongings simultaneously, while only one person can pass the screening at the same time. Depending on whether the passenger travels within or outside the Schengen area, he has to pass through the passport control before he can proceed to the gate of the corresponding flight.

Boarding begins $60 \mathrm{~min}$ and ends $20 \mathrm{~min}$ before departure $[26,27]$. If at this time there are still passengers in the queue for boarding, the boarding desk remains open until there are no more passengers in the queue. Passengers arriving at the gate after boarding has closed missed their flight and will leave the airport.

\subsection{Implementation into AnyLogic}

To implement the model we chose the commercial, java-based simulation software Anylogic. In this software, the user is provided with different simulation libraries and function blocks that can be customized using java code. In this case, we use the Process Modeling Library to implement the model of the airport via a flowchart. The characteristics and the behavior of the passengers are specified via a database.

The passengers are generated by a source block at the terminal entrance according to their arrival time at the airport stored in the database. Sink blocks are used in all situations where passengers are removed from the simulation. This is the case either if a passenger has passed through the boarding process or if the passenger arrives too late at check-in or boarding so that the counters already have been closed.

Check-in, security check, passport control and boarding are implemented as service stations. It is possible to specify the duration and the available number of service stations. Process stations are modeled as a single queue multiple server system i.e. only one passenger can be handled at a time and passengers have to wait in a queue for a free service station. The prioritization of the passengers in the queue is based on the first-in first-outprinciple. The check-in is implemented as common check-in. This means that all counters of an airline or an alliance are available to the passenger for check-in independently of their flight destination. Due to the normally high number of airlines at medium-sized airports we implemented three different groups of airlines for the check-in. The airlines are assigned to these three groups based on the number of passengers on the reference day. It should be noted that the type of airline is neglected and only the number of passengers is considered for the classification.

Passengers arriving at the airport before the check-in counters have been opened pass through a waiting loop in which they check every minute whether the check-in counters are open. As soon as the corresponding counter opens, they leave the waiting loop and join the queue. Passengers arriving at the check-in counter after the counters have been closed are removed from the simulation by a sink block. Boarding is implemented for each flight at a separate boarding counter. The procedure regarding queues and delayed passengers is identical to the check-in process. The number of counters for boarding is derived from the number of flights for which boarding takes place at the same time preventing an overlap of two flights at the same gate.

To divide passengers according to specific criteria, that are set in the database, e.g. into Schengen and nonSchengen passengers, we use select output blocks. In Fig. 2, the sink or source blocks are represented by ellipses. SelectOutput devices are illustrated by diamonds and the service stations by hexagons.

\subsection{Data input}

The results of our simulation are highly depended on the used input data. Due to a lack of information open to the public and privacy policies, we cannot rely on precisely measured data from airports. All the data used are taken from literature references. However, real data may be used by the airports themselves to conduct studies using our methodology. In the following, we divide the input data into fixed and process parameters. Fixed input parameters represent the effects of the airport 


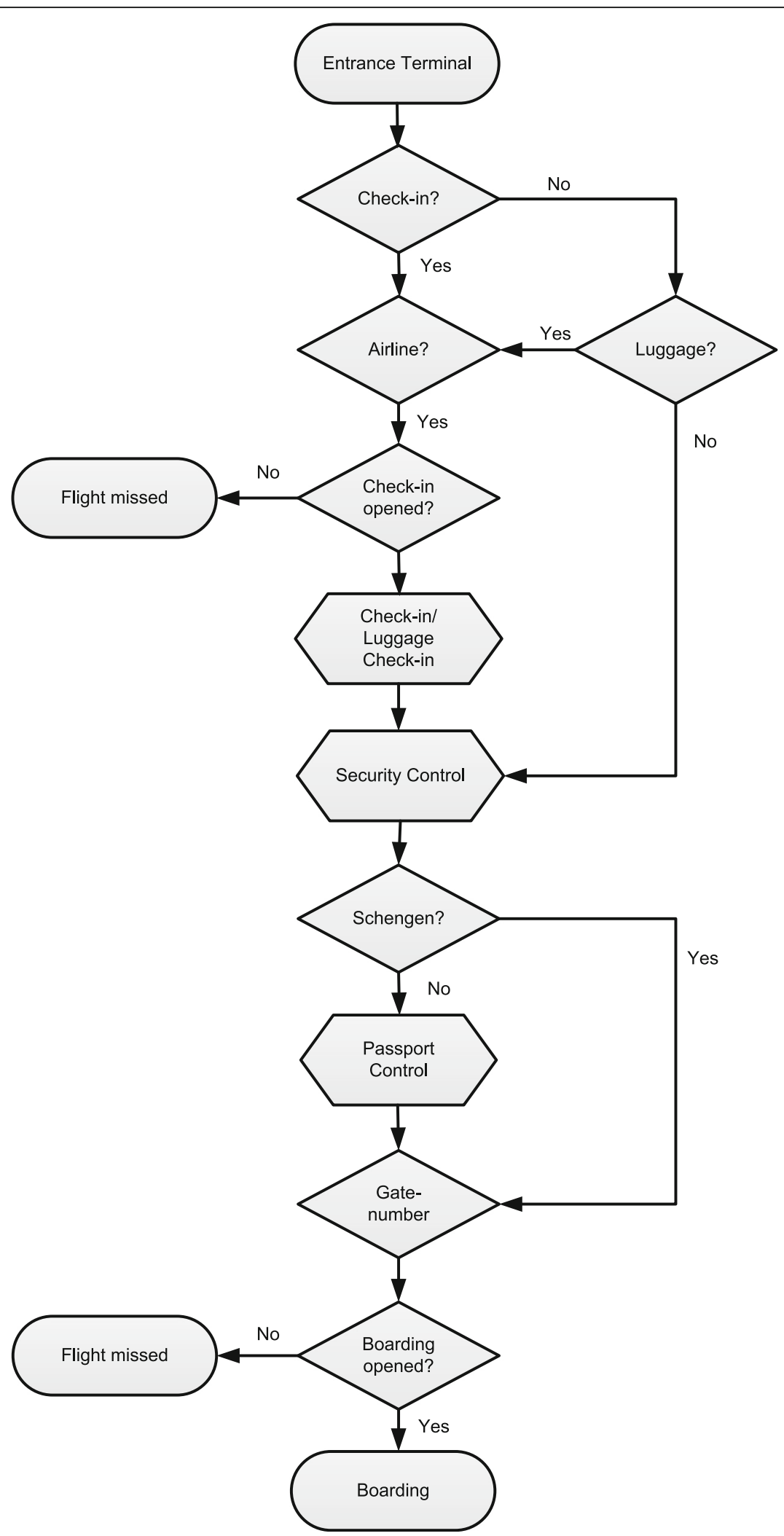

Fig. 2 Simulation logic for the departure processes. Ellipses show situations where passengers enter and leave the airport, service stations are represented by hexagons and diamonds outline chance nodes, that allocate passengers according to different criteria and probabilities 
environment on the model and can be hardly or not changed at all. The process parameters indicate the number of service stations at the passenger handling processes.

The output data illustrate the influence of the variation of the input data on the investigated issue. It always depends on the question, which parameters are considered and should be evaluated. The input and output data considered in this investigation are shown in Fig. 3.

The flight plan describes at which times the aircrafts will take off and land at the airport. The flight plan for the simulation is based on the flight plan for CologneBonn Airport dated 19.11.2018. This day was chosen randomly, because there is no information available regarding flight plans on peak days. The flight plan for the model contains the flight number of the respective flight, the destination (IATA code), the departure time, the operating airline (ICAO code) and the aircraft type that is used. The maximum number of seats available depends on the type of the aircraft and its configuration. This information is available via the public information provided by the airline. In average, the flights are not booked out. The SLF indicates the average occupancy rate of the seats available. In 2017, the average SLF in Germany was $77.6 \%$ [28]. The number of passengers generated in the model for the respective flights are derived from this SLF. Thus, the total number of passengers on the reference day is 16,136 assigned to 123 flights of 17 airlines. About 25\% of the passengers are non-Schengen.

To receive valid results, it is necessary to determine when the passengers arrive at the airport and the time available to complete the processes necessary for departure. According to the study of Schultz carried out at Stuttgart Airport, the arrival distribution of passengers at the airport corresponds to a normal distribution with an expected value of $89 \mathrm{~min}$ before departure and a standard deviation of $26 \mathrm{~min}$ [29]. The probability density function of a normal distribution is given in eq. 2 [30].

$$
p(x)=\frac{1}{\sigma \sqrt{2 \pi}} e^{-\frac{1}{2}\left(\frac{x-\mu}{\sigma}\right)^{2}}
$$

Whether the passengers arrive at the airport alone or in a group depends on the transport medium that is chosen to travel to the airport. Passengers using a car normally arrive either alone or in a small group, while passengers using public transport arrive in a group of passengers. According to the ADV, $64 \%$ of the passengers are using the car, a taxi or a rental car to arrive the

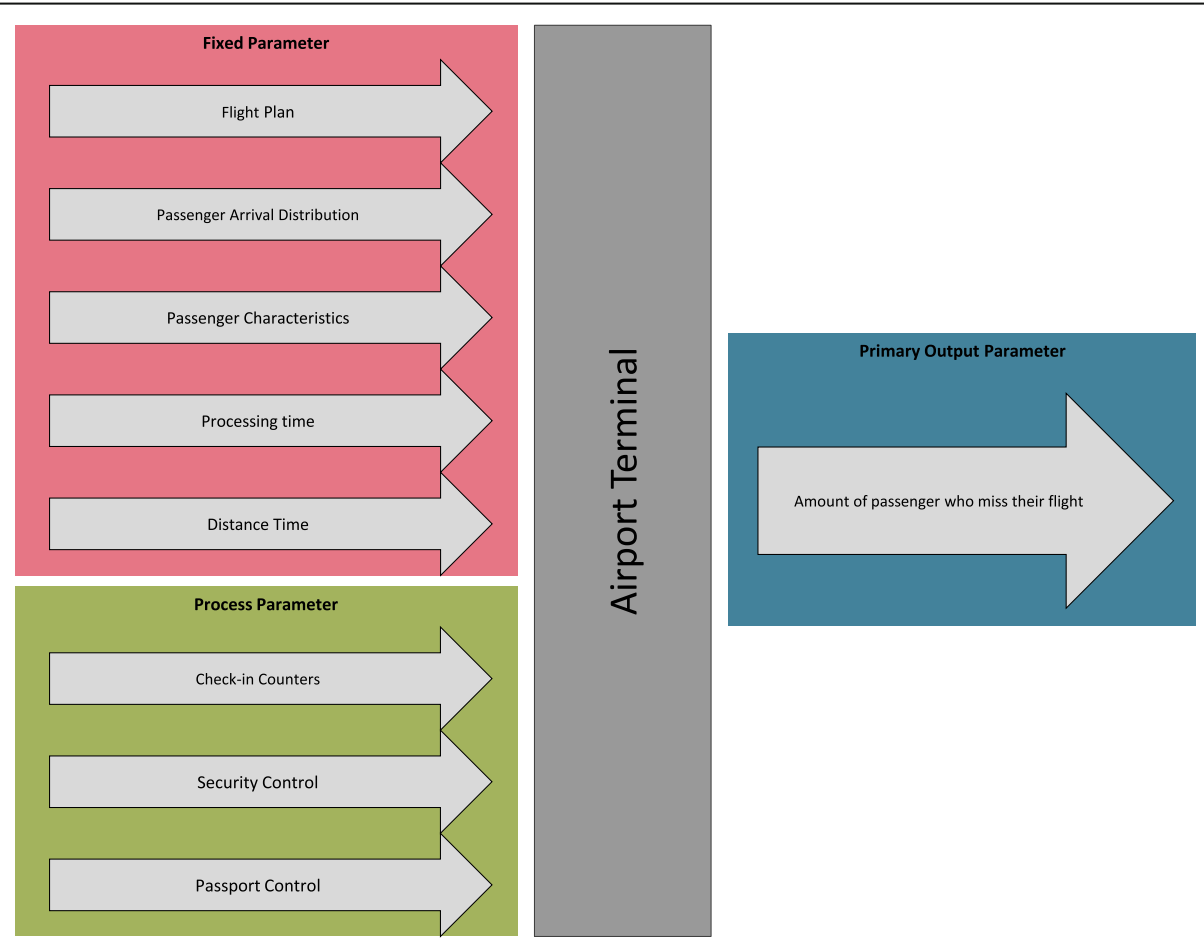

Fig. 3 Overview of the input and output parameters of the simulation. Input parameters are divided into fixed and process parameters. The red rectangle highlights the fixed parameters, which are constant or subject to a predefined probability distribution. The green rectangle contains the process parameter. These indicate the number of available counters at the process stations. The output parameters are shown in the blue rectangle 
airport, while $36 \%$ of the passengers are using public transport such as trains or buses [31].

The arrival time at the airport can be determined with the help of the arrival distribution, the information about the use of the transport media and the departure time of the respective flights using a Flight Generator developed by the DLR. The time at which each specific passenger arrives at the airport is based on pseudorandom numbers within the specified parameters (arrival distribution, departure time and transport medium). In order to analyze the influence of the random numbers on the results, 5 different databases with the arrival times of the passengers were generated. All of them are based on the three specifications mentioned above. Figure 4 shows the arrival times of the passengers before departure for the five databases. The $\mathrm{x}$-axis indicates when the passengers arrive at the airport before departure. The y-axis represents the number of passengers. The bars indicate the number of passengers arriving at the airport cumulated over $10 \mathrm{~min}$. The results of the individual databases are represented by the different colors of the bars. It is demonstrated that the arrival times of the different databases do not differ remarkably. The distributions correspond to a normal distribution (cf. equation 2) and, as expected, the mean value for the arrival time at the airport is between 80 and 90 min before departure. Passengers do not arrive at the airport earlier than $150-170 \mathrm{~min}$ and later than $0-10 \mathrm{~min}$ before departure. For all databases the majority of the passengers arrive at the airport between one and two hours before departure. About $11 \%$ of passengers $(\bar{x}=11.3 \%$ with $\sigma=$
$0.9 \%)$ arrive at the airport more than $2 \mathrm{~h}$ before departure, while about $14 \%(\bar{x}=14.4 \%$ with $\sigma=0.6 \%)$ arrive at the airport less than $1 \mathrm{~h}$ and about $2 \%(\bar{x}=1.5 \%$ with $\sigma=0.2 \%$ ) less than $30 \mathrm{~min}$ before departure.

If these passengers still have to pass the check-in process, they have no chance to reach their flight, as the check-in counters close 30 to $60 \mathrm{~min}$ before departure, depending on the airline and the destination and also boarding takes place about 20-30 min before departure [32]. Passengers who do not board their flight despite having booked a flight ticket are referred as no-shows. There are two main reasons for no-shows. First, some passengers book flex-tickets, which enable to book and cancel a flight flexibly without incurring additional costs. Second, some passengers arrive too late at the airport, for example due to traffic jams or the delay of public transport. The amount of the no-show rate depends on the airline and the route $[33,34]$. Within the model, the no-show rate is depicted by passengers arriving at the airport so late that the service stations of check-in or boarding are already closed so that they miss their flight. The no-show rate for this model is about $2 \%$.

In addition to the different arrival times of passengers at the airport, there are other passenger characteristics that are relevant for passenger handling. For example, we have to differentiate, whether the passenger has already checked-in online before arrival, or whether he has to check-in at the airport counter. Furthermore, some passengers travel with hand luggage only, while others have to check-in their baggage at the counter. According to a study by the aviation IT company SITA,

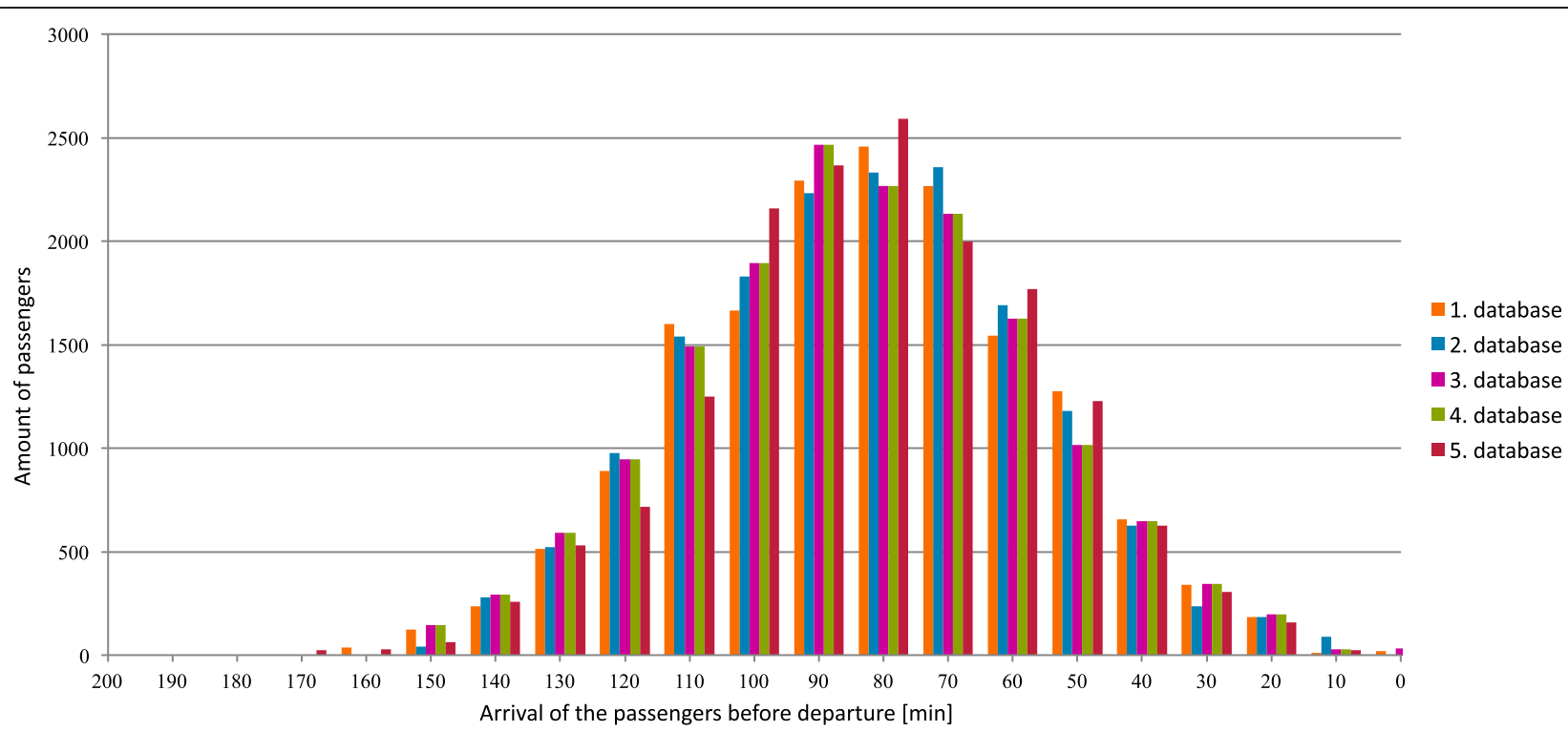

Fig. 4 Arrival distribution of passengers before departure of their flight in the five different databases. The bars represent the number of passengers, each accumulated over $10 \mathrm{~min}$. The arrival distribution in all databases corresponds to an expected value of 89 min before departure with a standard deviation of $26 \mathrm{~min}$ 
$39 \%$ of the passengers use online check-in, while $61 \%$ of the passengers still check-in at the airport counters [35]. Regarding baggage check-in we distinguish between passengers travelling within or outside Europe. Many airlines include baggage in the fares for flights outside of Europe. Thus, for this model we assume that all passenger with destinations outside of Europe travel with baggage. According to a study by Ryanair, passengers traveling within Europe carry baggage with a probability of $20 \%$ [36]. The different passenger characteristics are summarized in Table 1 . These characteristics are distributed randomly to the passengers with respect to the given percentage. To generate random numbers Mersenne Twister algorithm (MT 19937) is used [37]. In order to analyze the impact of this distribution, we use 5 different databases, as mentioned for the arrival times. The aim of the different databases is to examine that the changes of the output parameters result from a variation of the process parameters and not from random fluctuations within the fixed input parameters.

The processing time at the different process stations depends on the characteristics of the passengers, the conditions and the equipment available at the airport and the working methods of the personnel. Therefore, the processing time is subject to fluctuations. According to the study of Schultz at Stuttgart Airport, the duration of a check-in with or without baggage check-in is normal distributed (cf. equation 2) with an expected value of $58 \mathrm{~s}$ and a standard deviation of $25 \mathrm{~s}$ [29]. The security control is divided into three sub processes: disposal of personal belongings, security control of the person and the hand luggage and reception of personal belongings. The respective times are based on measurements carried out at Hamburg Airport as part of the project Critical Parts [38]. The time for depositing personal belongings varies between 0 and $81 \mathrm{~s}$ with a mode of $21 \mathrm{~s}$. Therefore, these times are subject to a triangular distribution. The probability density function $\mathrm{p}(\mathrm{x})$ is given in eq. 3 [30].

Table 1 Overview of the different passenger characteristics used for the simulation model

\begin{tabular}{ll}
\hline Passenger characteristics & Distribution \\
\hline Arrival distribution of the passenger at the airport [min] & $\mu=89 ; \sigma=26$ \\
Transportation mode (own car /public transport) [\%] & $64 / 36$ \\
Online check-in / counter check-in [\%] & $39 / 61$ \\
Baggage check-in within EU [\%] & 20 \\
Baggage check-in outside EU [\%] & 100 \\
Non-Schengen passengers [\%] & 25 \\
\hline
\end{tabular}

$$
p(x)=\left\{\begin{array}{cc}
\frac{2(x-a)}{(b-a)(c-a)} & \text { for } x \in[a, c] \\
\frac{2(b-c)}{(b-a)(b-c)} & \text { for } x \in[c, b] \\
0 & \text { else }
\end{array}\right.
$$

The security check consists of passing through a metal detector or a body scanner and, if necessary, a post control executed by the security stuff. The process time varies between 2 and $169 \mathrm{~s}$ with a mode of $43 \mathrm{~s}$ (cf. equation 3). The reception of personal belongings and, if necessary a baggage post inspection, takes between 0 and $260 \mathrm{~s}$ with a mode of $22 \mathrm{~s}$ (cf. equation 3 ). The passport control is uniform distributed and takes between 50 and $60 \mathrm{~s}$ [39]. The probability distribution function of the uniform distribution is given in eq. 4 [30].

$$
p(x)=\left\{\begin{array}{lr}
\frac{1}{(b-a)} & \text { for } a \leq x \leq b \\
0 & \text { else }
\end{array}\right.
$$

The duration of the boarding process is normal distributed with an expected value of $5 \mathrm{~s}$ and a standard deviation of $2 \mathrm{~s}$ (cf. equation 2). An overview of the process times used for this model is included in the Table 4 in Appendix.

The time the passengers need to cover distances ( $t$ ) can be calculated via the formula of uniform movement, dependent on the distances between the process stations (s) and the speed of the movement of the passengers (v) (cf. equation 5). The speed of movement distinguishes between people and is given in the form of a probability distribution. Moreover, the distances the passengers have to cover between the stations also vary, e.g. depending on the entrance a passenger enters the terminal or the gate the boarding takes place. For this reason, the times the passengers need between the process stations are not constant. The level of the variation depends on several variables. First, on the uncertainty of the distance that has to be covered, and second, on a different walking speed of the people. Quantities that depend on several variables with uncertainties can be determined with the help of Gaussian error propagation [40]. Thus, the variation of the travel times can be calculated with eq. 6 .

$$
\begin{aligned}
& t=\frac{s}{v} \\
& d t=\sqrt{\left(\frac{1}{v}\right)^{2} * d s^{2}+\left(\frac{s}{v^{2}}\right)^{2} * d v^{2}}
\end{aligned}
$$

According to Weidmann of the ETH Zurich, the speed of human movement is $1.34 \mathrm{~m} / \mathrm{s}$ with a standard deviation of $0.26 \mathrm{~m} / \mathrm{s}$ [41]. The distances between the process stations depend on the structure and the size of the respective airport. In this model, the distances are 
Table 2 Distances between the process stations and the resulting times the passengers need tocover these distances (delay times) for the model. The distances as well as the delay times are indicated by the mean value and the standard deviation

\begin{tabular}{llll}
\hline Route & & Distance [m] & Delay Times [s] \\
\hline Entrance Terminal - & Check-in Counter & $46 \pm 26$ & $34 \pm 20$ \\
Entrance Terminal - & Security Control & $153 \pm 77$ & $114 \pm 62$ \\
Check-in Counter - & Security Control & $153 \pm 77$ & $114 \pm 62$ \\
Security Control - & Passport Control & 90 & $67 \pm 13$ \\
Security Control - & Gate & $106 \pm 42$ & $79 \pm 35$ \\
security Control - & Gate & $42 \pm 15$ & $31 \pm 13$ \\
\hline
\end{tabular}

based on the structure of the airport Cologne-Bonn. An overview of the distances between the process stations and the resulting times is shown in Table 2. Appropriate data for other airports can be calculated the same way with adjusted distances.

The process parameters are represented by the number of service stations available for check-in, security and passport control. Unlike the fixed input parameters, these can be adapted by the airport operator or the airlines to the occupancy rate or available resources (e.g. personnel or power availability). For this model, we assume that the number of opened service stations at the passenger handling process stations is static throughout the day and must be determined at the beginning of the day. Therefore, it is not possible to customize the number of opened service stations to fluctuating passenger volumes during the course of the day.

The range in which we can vary the process parameters depends on the number of the counters at the service stations available at Cologne-Bonn Airport. For check-in a maximum number of 86 counters, for security checks a maximum number of 26 counters and a maximum number of 6 counters for passport checks are available. We assume that all processes have to continue in order to maintain flight operations. Therefore, at least one service station must be available at each process. As explained above available check-in counters are divided into three groups by the number of passengers the group has to handle at the reference day. The first group only handles passengers from Eurowings, which corresponds to $50 \%$ of the passenger volume. The second group handles $28 \%$ of the passenger volume which consists of clients from Lufthansa and Ryanair. The last group checksin all passengers from other airlines.

As output data we investigate the percentage of passengers missing their flight. This parameter is used to evaluate how the variation of the process parameters affects the proportion of passengers who do not reach their flight. With this data we can calculate a resulting average SLF for the flights. From a certain SLF it is no longer economically sensible to operate the flight, as the revenues from the flight tickets are lower than the variable operating costs. This seat load factor is called break-even seat load factor (break-even SLF) [42]. In recent years, the break-even SLF has averaged 66\% [43].

\section{Simulation results}

Initially, we assume that there is no disruption at the airport and that the maximum number of all service stations is available. When the disruption occurs, less power is available at the airport for passenger handling. Therefore, the number of service stations must be reduced. To analyze the impact of the different processes, we only reduce the number of service stations at one process until so many passengers miss their flight that the resulting SLF falls below the break-even SLF. This procedure is performed five times with each of the different databases. The resulting SLF is averaged over the runs. The standard deviation indicates the variance of the SLF induced by the five databases.

First test runs showed that a reduction of the number of check-in counters initially had little effect on the number of passengers who did not reach their flight. Therefore, we determined the reduction intervals for check-in to 10 counters. From a reduction to 46 counters we perceive greater effects. Thus, we halved the reduction intervals. In addition, during this runs it became clear that the SLF falls below the break-even SLF with a number of 6 check-in counters, 19 security checks or 2 passport checks. Therefore, we only reduce the available counters at the respective process stations up to this limit. In total, we executed 520 simulation runs for each of the five data bases. Figure 5 shows the simulation space with the considered parameter, the mean values and the standard deviations of the results.

The axes show the number of security checks, checkin counters and passport control. The points represent the coordinates of the parameter space for which we performed simulation runs. The SFL results from the corresponding combinations of the service counters at the three processes. In Fig. 5 (a) the mean SLF is colorcoded. The legend next to the parameter space shows how the color is related to the average SLF of the flights over the day. The blue dashed line indicates the maximum SLF possible in the model of $77.6 \%$. The red dashed line represents the break-even SLF of $66 \%$. Bright dots indicate a high SLF. The darker the color, the lower is the SLF. White or yellow dots indicate that the mean SLF is above the break-even SLF of $66 \%$. Dark brown and black dots indicate that the mean SLF is below the break-even SLF. For light brown dots, the SLF is close to the break-even SLF. 


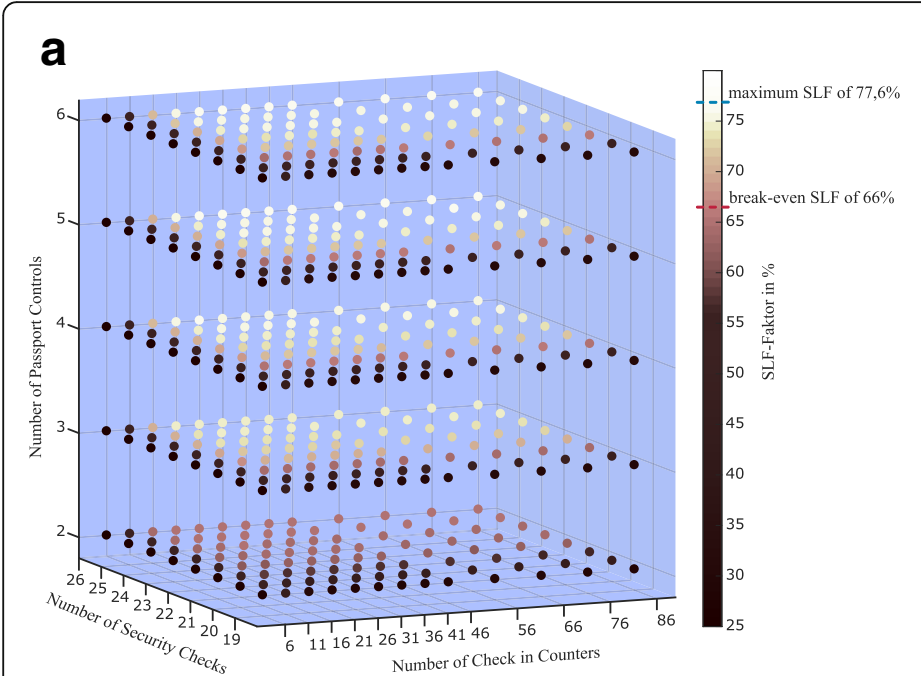

b

Fig. 5 Representation of the considered parameter space with the number of service counters where we carried out simulation runs. We determined the mean values and the standard deviations of the results of the runs with the five different databases. These are shown in Fig. 5 (a) and 5 (b). a Representation of the mean values. $\mathbf{b}$ Representation of the standard deviations

If all service stations of the three processes are available, the SLF is between $70 \%$ and $76 \%$ and therefore above the break-even SLF. Figure 5 (a) shows that initially the SLF does not change notable when the check-in counters are reduced. From a number of 16 check-in counters onwards we can see a greater change in the SLF. As of a number of 11 available check-in counters the SLF falls below the break-even SLF. Reducing the number of security checks down to 21 does not lead to a lowering of the SLF below break-even level. With a number of 19 and 20 available security checks, the SLF falls below the breakeven SLF. If we reduce the number of service stations at the passport control, no notable influence on the SLF can be detected. Only at a number of 2 service counter we can perceive a change in the SLF. Here the SLF has a value slightly lower than the breakeven SLF.

Figure 5 (b) shows the difference of the results of the simulation runs with different databases in form of the standard deviation. The coloring of the dots outlines the percentage standard deviation. Dark dots represent a high standard deviation while bright dots symbolize a low standard deviation. The maximum deviation of the results for all parameter combinations is less than $6 \%$. With a high number of all service stations at the three processes, the results of the simulation runs differ by only less than $1 \%$. Thus, we can exclude the differences in the databases, e.g. the distribution of arrival times, as the cause of the decreasing SLF.
The results show that it is possible to reduce the number of check-in counters to 26 without a noticeable increase in the number of passengers missing their flight. If a system failure necessitates a further reduction in check-in resources, these can be reduced to 21 or 16 counters. However, we must expect that the quota of passengers who miss their flight will increase up to $5 \%$. Based on the results of this simulation study, a further reduction in the number of check-in counters is not recommended, because otherwise the proportion of occupied seats in the aircraft would fall below the breakeven SLF.

Already a reduction of one counter at the security control has a noticeable effect on the number of passengers not reaching their flight. However, if the situation at the airport makes it unavoidable to reduce security control resources, we can reduce them to 22 available service stations. With a number of 21 service stations at the security control, the average proportion of occupied seats in the aircraft is just below the break-even SLF. Since the random fluctuations of the SLF are between $2 \%$ and $3 \%$, we cannot clearly determine whether the break-even SLF is slightly exceeded or undercut. If the number of service stations undercuts 21 , the number of passengers who do not reach their flight increases sharply. Therefore, we do not advise a reduction of the number of security checks to less than 21.

According to the simulation results, a reduction of passport control to 3 service stations is possible without a noticeable increase in delayed passengers. With a 
reduction of resources to 2 passport controls so many non-Schengen passengers miss their flight that the SLF, both in terms of the number of non-Schengen passengers and the total number of passengers, falls below the break-even SLF.

Since no information is available regarding the power consumption of the respective processes, we cannot make a statement regarding an optimal combination of the service stations. However, the results of the simulation provide an aid in deciding which variations of the service stations are considered as uncritical, critical or insufficient with regard to a functioning flight operation. This overview of the results is shown in Table 3.

If less power is available at the airport due to a system malfunction, we initially recommend reducing the number of check-in and passport control service stations. Up to 26 service stations at the check-in or 4 at the passport control, the reduction has only a minor effect on the number of passengers missing their flight. If a further reduction in resources is necessary, the number of service stations at the security control can also be reduced. A reduction to fewer than 16 counters for check-in, 21 service stations for security or 3 for passport control should be avoided. This would lead to so many passengers who do not reach their flight that the SLF would fall under the break-even SLF.

\section{Conclusion and outlook}

As part of the research project "Airport Blackout", we developed a method to investigate the possibility to save resources in passenger handling processes at the airport terminal without interrupting flight operations. The aim is to provide decision support for an improved reaction to system failures, for example during power shortages. With the data of the study responsible actors are able to decide which and how many passenger handling service stations can be dispensed without interrupting flight operations.

The results of the simulation show that it is possible to reduce the number of service stations at the check-in from a maximum number of 86 to 26

Table 3 Assessment of the number of available service stations at passenger handling processes as uncritical, critical and insufficient to maintain flight operations

\begin{tabular}{llll}
\hline & Uncritical & Critical & Insufficient \\
\cline { 2 - 4 } service station for check-in & $86-26$ & $21-16$ & $11-6$ \\
service stations for security check & 26 & $25-21$ & $20-19$ \\
service stations for passport control & $6-4$ & 3 & 2 \\
\hline
\end{tabular}

counters and at the passport control from a maximum number of 6 to 4 service stations without affecting the number of delayed passengers. The security control is the bottleneck in the passenger handling process. We have to expect a reduction in the average SLF as soon as the number of service stations at the security check is reduced. With a high number of service stations at the security check, these effects are within a few percentages. Therefore, a small reduction in the number of service stations at the security check is possible without interrupting flight operations. In view of the break-even SLF, a reduction in resources at the check-in below 16 counters, at the security check below 21 and at the passport control below 3 service stations should be avoided.

As the input data is based on several general assumptions and data from Cologne-Bonn Airport at a specific day, these results are not directly transferrable to all airports. Due to the standardization of procedures at international airports, both, the methodology and the simulation model, are transferable. These may be adapted using data of various airports and therefore represent a generally applicable instrument for decision support.

Since the system of the airport terminal is very complex, we made several simplifications and assumptions for the structure of the simulation. The extent to which for example an introduction of spatial boundaries of the airport terminal into the simulation model, a variable adaptation of the service stations to a fluctuating passenger volume in the course of the day or different flight schedules have an influence on the results of this investigation, should be investigated in more detail and represent a research topic of further projects. In addition, in this project we only considered passenger handling processes for departure. The possibility to save resources in other processes at the airport terminal, such as baggage handling, should also be investigated in further studies. Thus, the results of this study provide a first approach for improving the reactive measures in the event of system failures at airports, which have to be examined and specified in more detail in further projects. Then the results will be applied not only to system failures in the form of power shortages at the airport, but also for example, to personnel shortages, technical problems at individual service stations or a partial IT failure. Whenever resources for passenger handling are available to a limited extent, the findings can support the responsible actors in their decision making on how to use the available resources in an optimal manner and whether they are sufficient to maintain flight operations. 


\section{Appendix}

Table 4 Overview of the core equations used in the model

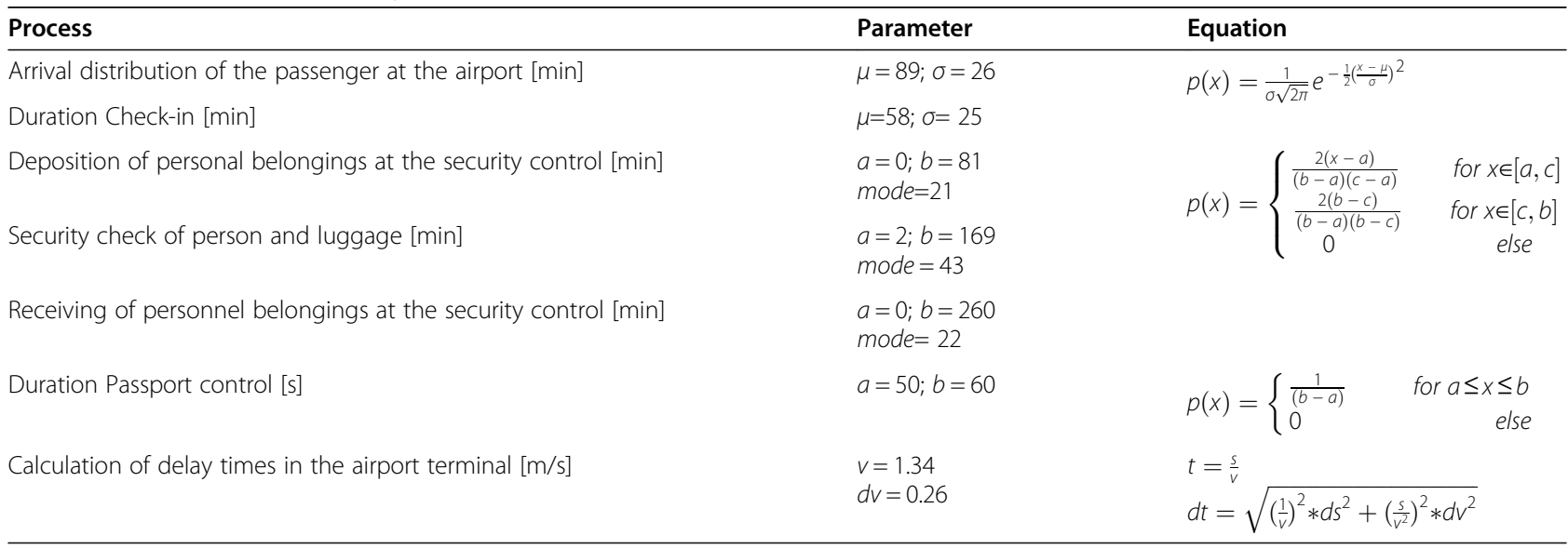

\section{Acknowledgements}

No applicable.

\section{Authors' contributions}

Conceptualization: LMB, AP and TK; Data curation: LMB; Funding acquisition: $\mathrm{MH}$; Investigation: LMB, AP, TK; Supervision: AD; Writing: LMB, Writing review \& editing: $A P, T K, A D, M H$. The authors read and approved the final manuscript.

\section{Funding}

The research is part of the project "Blackout at Airports "and was funded by DLR's programmatic research funds as part of the Helmholtz Association. Open Access funding enabled and organized by Projekt DEAL.

\section{Availability of data and materials}

The datasets used and analyzed during the current study are available from the corresponding author on reasonable request.

\section{Competing interests}

The authors declare that they have no competing interests.

\section{Author details}

'German Aerospace Center (DLR), Institute of Air Transport and Airport Research Lilienthalplatz 7, 38108 Braunschweig, Germany. ${ }^{2}$ German Aerospace Center (DLR), National Experimental Test Center for unmanned Aircraft Systems, Harzstraße 1, 39444 Hecklingen, Germany. ${ }^{3}$ German Aerospace Center (DLR), Program Coordination Defence \& Security Research, Linder Höhe, 51147 Köln, Germany.

Received: 20 March 2020 Accepted: 25 September 2020

\section{Published online: 06 October 2020}

\section{References}

1. Mensen, H. (2013). Handbuch der Luftfahrt, (2nd ed., ). Berlin Heidelberg: Springer Vieweg.

2. Stegner, C., \& Meinberg, U. (2015). Leistungs- und Qualitätsmessung für einen passagierorientierten Umgang mit Betriebsstörungen im Luftverkehr. BTU Cottbus-Senftenberg Dissertation. https://d-nb.info/1114283886/34. Accessed 2 Oct 2020.

3. EASA (2019). Easy access rules for aerodromes (regulation (EU) no. 139/2014) Retrieved from: https://www.easa.europa.eu/sites/default/files/dfu/Easy_ Access_Rules_for_Aerodromes-May2019_0.pdf. Accessed: 05 Mar 2020.

4. Herling, S., \& Polders, I. B. (2017). Deutschland ist nicht Atlanta: Deutsche Flughäfen bei Stromausfall bestens vorbereitet Retrieved from: https://www. adv.aero/wp-content/uploads/2017/12/ADV-PM-24-2017-Deutschland-istnicht-Atlanta-\%E2\%80\%93-Deutsche-Flugh\%C3\%A4fen-bei-Stromausfallbestens-vorbereitet.pdf. Accessed: 12 Jan 2019.
5. Mosel, L. (2018). Hamburger Flughafen nach Stromausfall geräumt Passagiere beklagen chaotische Zustände Retrieved from: https://www.welt. de/vermischtes/article176922234/Hamburger-Flughafen-nach-Stromausfallgeraeumt/-Passagiere-beklagen-chaotische-Zustaende.html. Accessed: 16 Feb 2019.

6. Koch, T., Deutschmann, A., \& Moller, D. P. F. (2019). Using process-orientedgrouping and linear algebra to optimize resource management. https://doi. org/10.1109/ICNSURV.2019.8735110.

7. Gruno, G., Diglio, A., Genovese, A., \& Piccolo, C. (2018). A decision support system to improve performances of airport check-in services. https://doi.org/10. 1007/s00500-018-3301-z.

8. Lalita, T. R., Manna, D. K., \& Murthy, G. S. R. (2020). Mathematical formulations for large scale check-in counter allocation. https://doi.org/10.1016/j.jairtraman. 2020.101796.

9. Fraport, (n.d.). Passagierflusssimulationen am Frankfurter Flughafen, The AnyLogic Website. Retrieved from: https://www.anylogic.de/simulation-ofthe-frankfurt-airport/. Accessed: 05 Nov 2019.

10. Curcio, D., Longo, F., Mirabelli, G., \& Pappoff, E. (2007). Passengers' flow analysis and security issues in airport terminals using modeling \& simulation. https://doi.org/10.7148/2007-0374.

11. Asmer, L., Popa, A., Koch, T., Deutschmann, A., \& Hellmann, M. (2019). Secure Rail Station - Research on the effect of security checks on passenger flow. https://doi.org/10.1016/j.jrtpm.2019.04.002.

12. Pao-Yen Wu, P., \& Mengersen, K. (2012). A review of models and model usage scenarios for an airport complex system. https://doi.org/10.1016/j.tra.2012.10.015.

13. Solak, S., Clarke, J. P., \& Johnson, E. L. (2009). Airport terminal capacity planning. https://doi.org/10.1016/j.trb.2009.01.002.

14. Brevilacqua, M., \& Ciarapica, F. E. (2010). Analysis of check-in procedure using simulation: A case study. https://doi.org/10.1109/IEEM.2010.5674286.

15. Appelt, S., Batta, R., Lin, L., \& Drury, C. (2007). Simulation of passenger check-in at medium-sized airport. https://doi.org/10.1109/WSC.2007.441979229.

16. Manataki, I. E., \& Zografos, K. G. (2009). A generic system dynamics based tool for airport terminal performance analysis. https://doi.org/10.1016/j.trc.2009.02.001.

17. Chawdhry, P. K. (2009). Risk modelling and simulation of airport passenger departure process. https://doi.org/10.1109/WSC.2009.5429244.

18. Yeh, C.-H., \& Kuo, Y. L. (2003). Evaluating passenger services of Asia-Pacific international airports. https://doi.org/10.1016/S1366-5545(02)00017-0.

19. Gkritza, K., Niemeier, D., \& Mannering, F. (2006). Airport security screening and changing passenger satisfaction: An exporatory assessment. https://doi.org/10. 1016/j.jairtraman.2006.03.001.

20. Martonosi, S. E. (2011). Dynamic server allocation at parallel queues. https:// doi.org/10.1080/0740817X.2011.564602

21. Ilic, M. D., \& Zaborsky, J. (2000). Dynamics and control of large electric power systems. Chichester: Wiley-IEEE Press.

22. Koch et al. (2020). Smart technologies as a thread for critical infrastructures. In Smart technologies by Akhilesh, K.B, Möller, D.P.F.

23. ICAO (2017). International standards and recommended practices annex 9.

24. European Commission (2015). Regulation (EU) no 2015/1998. 
25. Flughafen Köln-Bonn (2019). Check-in Retrieved from: https://www.reisenexperten.de/reiseinformationen/flughafeninfos/flughafen-kolnbonn/checkin-682/. Accessed: 19 Jan 2019.

26. KLM (2019). Zeitvorgaben Retrieved from: https://www.klm.com/travel/at_de/ prepare_for_travel/at_the_airport/requirements/index.htm. Accessed: 19 Jan 2019.

27. Iberia (2019). Das boarding und die Serviceleistungen an Bord Retrieved from: https://www.iberia.com/de/annahme-schlusszeiten/. Accessed: 12 Jan 2019.

28. DFS (2017). Luftverkehr in Deutschland: Mobilitätsbericht 2017 Retrieved from: https://www.dfs.de/dfs_homepage/de/Presse/Publikationen/ Mobilitaetsbericht2017_Web.pdf. Accessed: 03 Mar 2020.

29. Schultz, M. (2010). Entwicklung eines Individuenbasierten Modells zur Abbildung des Bewegungsverhaltens von Passagieren im Flughafenterminal Dissertation, TU Dresden

30. Forbes, C., Evans, M., Hastings, N., \& Peacock, B. (2011). Statistical distributions. https://doi.org/10.1002/9780470627242.

31. ADV (2018). Airport travel survey 2018: Zahlen, Fakten, trends Retrieved from: https://www.adv.aero/wp-content/uploads/2016/02/Airport-Travel-Survey-2 018_Brosch\%C3\%BCre.pdf. Accessed: 05 Mar 2020.

32. Asam, S. (2018). Wie viel Zeit muss ich am Flughafen einplanen? Retrieved from: https://www.merkur.de/reise/viel-zeit-muss-eigentlich-flughafeneinplanen-zr-8697182.html. Accessed: 01 Mar 2019.

33. Lawrence, R. D., Hong, S. J., \& Cherrier, J. (2003). Passenger-based predictive modeling of airline no-show rates. https://doi.org/10.1145/956750.956796.

34. Horny, T. (2017). Warum gibt es no-shows? Retrieved from: https:/www. focus.de/reisen/flug/tid-8118/ueberbuchungen_aid_145399.html Accessed: 01 Mar 2019.

35. Eiselin, S. (2018). Check-in: Warum Passagiere noch immer Schalter nutzen Retrieved from: https://www.aerotelegraph.com/check-in-warum-passagierenoch-immer-schalter-nutzen. Accessed: 19 Jan 2019.

36. Hase, T. (2018). Nur Handgepäck dabei? Darum sollten Sie sich beim boarding schnell anstellen Retrieved from: https://www.merkur.de/reise/fliegen-nurmit-handgepaeck-zr-8422049.html. Accessed: 19 Jan 2019.

37. Office Support. (n.d.) Retrieved from: https://support.office.com/en-US/ article/RAND-function-4CBFA695-8869-4788-8D90-021EA9F5BE73. Accessed: 12 June 2020

38. Popa, A., \& Ster, J. (2014). Port security: Simulation alternativer Kontrollstellen am Fährterminal in Rostock.

39. Kachlíková, M. (2011). Selbstbedienung bei Passkontrolle - neues E-gate auf Prager Flughafen Retrieved from: https://www.radio.cz/de/rubrik/tagesecho/ selbstbedienung-bei-passkontrolle-neues-e-gate-auf-prager-flughafen. Accessed: 19 Jan 2019

40. Papula, L. (2019). Mathematik für Ingenieure und Naturwissenschaftler Anwendungsbeispiele: 22 Aufgabenstellungen mit ausführlichen Lösungen, (8th ed., ). Wiesbaden: Springer Fachmedien Wiesbaden GmbH.

41. Weidmann, U. (1992). Transporttechnik der Fussgänger: Transporttechnische Eigenschaften des Fussgängerverkehrs, Literaturauswertung. https://doi.org/10. 3929/ethz-a-000687810

42. Butler, G. F., \& Keller, M. R. (2000). Handbook of airline operations, (1st ed., ). Aviation Week. New York: McGraw-Hill Company.

43. Avjobs. (2010). Airline Economics. Retrieved from: https://www.avjobs.com/ history/airline-economics.asp. Accessed: 07 Feb 2019

\section{Publisher's Note}

Springer Nature remains neutral with regard to jurisdictional claims in published maps and institutional affiliations.

\section{Submit your manuscript to a SpringerOpen ${ }^{\circ}$ journal and benefit from:}

- Convenient online submission

- Rigorous peer review

- Open access: articles freely available online

- High visibility within the field

- Retaining the copyright to your article

Submit your next manuscript at $\boldsymbol{\nabla}$ springeropen.com 